\title{
Periodontal manifestations of Langerhans cell histiocytosis: a systematic review
}

\author{
Julia C. Difloe-Geisert ${ }^{1}$ (D) Selina A. Bernauer ${ }^{1} \cdot$ Noémie Schneeberger $^{1} \cdot$ Michael M. Bornstein $^{2} \cdot$ Clemens Walter $^{1}$
}

Received: 21 January 2021 / Accepted: 3 March 2021 / Published online: 22 March 2021

(C) The Author(s) 2021

\begin{abstract}
Objectives To explore the evidence of periodontal manifestations and treatment modalities in patients with Langerhans cell histiocytosis (LCH).

Material and methods A systematic literature search was performed and the criteria for PRISMA and risk of bias assessment were applied. Human clinical studies ( $\geq 10$ patients) presenting patients with LCH and periodontal findings were considered for inclusion.

Results From 298 titles identified, six case series with a total of 1278 patients suffering from LCH were included. In these studies, oral symptoms were reported in a frequency ranging from 10 to $100 \%$. Overall, in 216 patients (17\%), oral symptoms were observed. Out of these patients, $49-100 \%$ demonstrated periodontal symptoms. The most common oral findings were pain, swelling, tooth loss/mobility, and bone lesions. Specific periodontal findings comprised varying frequencies of gingival ulcerations, increased pocket depths, and gingival bleeding. Treatment measures constituted of surgical curettage of bone lesions, soft tissue excision and/or tooth extractions, radiotherapy, systemic chemotherapy, or a combination of these approaches. Healing without recurrence of oral lesions was reported in most of the cases.

Conclusions The available evidence on periodontal manifestations in LCH patients is heterogeneous. Several oral and periodontal findings were reported and may occur as initial symptoms and/or at later stages of the disease.

Clinical relevance The dentist should be aware of possible oral involvement of systemic diseases such as $\mathrm{LCH}$, and these manifestations may mimic periodontal disease.
\end{abstract}

Keywords Langerhans cell histiocytosis $\cdot$ Systemic diseases $\cdot$ Oral manifestations $\cdot$ Periodontal diseases

\section{Introduction}

Langerhans cell histiocytosis (LCH; older term: histiocytosis $\mathrm{X})$ is defined as a rare disease with an estimated annual incidence of 5 to 9 cases per million in children $<15$ years of age [1-3], and 1 case per million in patients $>15$ years of age [4]. The disease can occur at any age; however, it is most

Julia C. Difloe-Geisert and Selina A. Bernauer shared first authorship.

Clemens Walter

clemens.walter@unibas.ch

1 Department of Periodontology, Endodontology and Cariology, University Center for Dental Medicine Basel (UZB), University of Basel, Mattenstrasse 40, 4058 Basel, Switzerland

2 Department Oral Health \& Medicine, University Center for Dental Medicine Basel (UZB), University of Basel, Basel, Switzerland commonly diagnosed during childhood [1]. A slight predominance was reported in males $[1,2]$.

$\mathrm{LCH}$ is characterized by an excessive proliferation and accumulation of histiocytes (also called Langerhans cells) in various tissues [5-7]. This proliferation can lead to an infiltration of organs, thereby replacing the normal organ structure. Up to now, the understanding of LCH pathogenesis is still incomplete; however, neoplastic and inflammatory stimuli may contribute to disease progression. Histopathologically, infiltrates of histiocytes are present, with variable numbers of eosinophils, plasma cells, lymphocytes, and/or multinucleated giant cells [8]. The clinical presentation of LCH depends on the involved organs; e.g., bone, skin, lymph system, liver, lung, hematopoietic system, and/or oral tissues can be affected. Secondary complications may occur, such as diabetes insipidus following infiltration of the hypophysis. Historically, LCH was classified into the three clinical subtypes eosinophilic granuloma, Hand-Schüller-Christian 
disease, and Letterer-Siwe disease [5]. A current approach is to categorize LCH into a single-system form, i.e., a single organ is affected, and a multi-system form, i.e., several organs are involved [8]. Organ involvement may influence the progression and severity of disease, and a more favorable prognosis exists in patients with a single-system form [9].

In the current classification of periodontal and peri-implant diseases and conditions of 2018, LCH is listed as a "disorder that may be associated with progressive loss of the alveolar bone and increased mobility of affected teeth" $[10,11]$. The strength of association was estimated as moderate. Therefore, the aim of the present systematic review was to assess the evidence of periodontal manifestations and treatment modalities in patients suffering from $\mathrm{LCH}$.

\section{Material and methods}

\section{Protocol}

The present systematic review was accomplished adhering to PRISMA (Preferred Reporting Items for Systematic Reviews and Meta-Analyses) criteria (Online Resource 1) [12, 13].

\section{Outcome measures}

The primary outcome measures were periodontal symptoms as assessed clinically or radiologically in patients suffering from LCH. Therapies applied and response of oral lesions to these therapeutic approaches were considered secondary outcome variables.

\section{Eligibility criteria}

Publications were considered eligible for inclusion in this systematic review if the following parameters were presented:

(1) Original in vivo study, i.e., randomized controlled clinical studies, controlled clinical studies, prospective or retrospective case series,

(2) Study performed in humans,

(3) Study including at least 10 patients [14],

(4) Diagnosis of LCH as primary disease,

(5) Presentation of healthy or diseased periodontal soft and hard tissues (i.e., gingiva and/or periodontal ligament and tooth-bearing parts of the alveolar bone), and

(6) Publication in English, French, or German language.

\section{Information sources and search}

The electronic databases MEDLINE and Embase via Ovid, Cochrane Central Register of Controlled Trials
(CENTRAL), and the gray literature (http://www.opengrey. eu) were searched for studies published up to January 13, 2021. The search protocols within the different databases were applied and validated as identically as possible (Online Resource 2). The following search terms were applied: "histiocytosis" OR "Langerhans cell" OR "eosinophilic granuloma" OR "Hand Schuller" AND "periodont*" OR "oral disease" OR "oral involvement" OR "oral lesion" OR "oral manifestation" OR "dental disease" OR "dental involvement" OR "dental lesion" OR "dental manifestation."

In addition, potentially relevant citations were harvested from the bibliographies of included studies and relevant reviews on the topic and examined for inclusion eligibility. The references resulting from the searches were entered in EndNote (Version X9, Clarivate Analytics, Philadelphia, PA, USA), and duplicates were removed.

\section{Study selection}

Screening of records was performed independently by two of the authors (S. A. B. and J. C. D.). Cohen's kappa score was calculated to assess inter-examiner agreement [15]. Eligibility assessment was performed through title and abstract analysis and secondly through full-text analysis (Fig. 1, Online Resource 3). From all studies of potential relevance, fulltexts were obtained for independent assessment by the reviewers. Any disagreement was resolved by discussion among the two authors. The software system for recording decisions was Microsoft Excel (Version 16.44, Microsoft Corporation, Redmond, WA, USA).

\section{Data collection process}

The following data of included studies were collected in duplicate by two independent reviewers using standardized data extraction files (Microsoft Word, Version 16.44, Microsoft Corporation, Redmond, WA, USA): characteristics of the study population, systemic manifestations, oral manifestations, treatment modalities, dropouts, duration, and results of follow-up oral examinations (Table 1).

\section{Frequency distribution of oral LCH manifestations}

A frequency distribution of each finding (e.g. involved organ systems and symptoms) was calculated and shown as an absolute number and percentage [22]. Findings not reported in any patient of a case series were interpreted as not investigated and excluded from the respective frequency calculations. Thus, the reference number of patients differed from finding to finding. The maximum possible reference number of patients was the sum of all included patients (if a finding was examined in all included case series). 
Fig. 1 Selection process for the studies included [12]
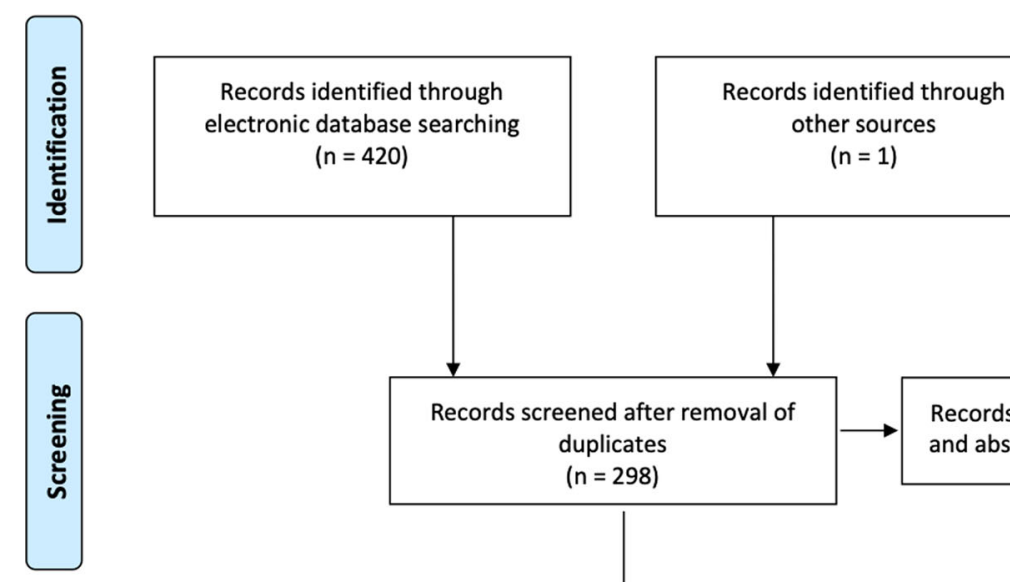

Records identified through

other sources

$(n=1)$
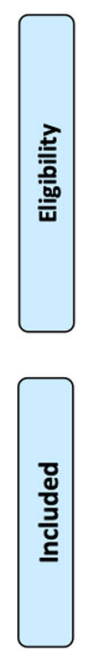

\section{Quality assessment of included studies}

The risk of bias of included studies was evaluated by the checklist from Moga et al. [23], including 18 items of the following topics: (a) study objective; (b) study population; (c) intervention and co-intervention; (d) outcome measure; (e) statistical analysis; and (f) results and conclusion (Online Resource 4). Considering the adequacy in the respective case series, the items were graded and the percentage of positively graded items was calculated [14, 24] (Online Resource 5).

\section{Results}

\section{Study selection}

A total of 298 studies were identified by electronic and hand search (Fig. 1). After title and abstract screening (inter-examiner Cohen's kappa score $=0.75$ ), the full texts of 19 studies were screened for possible inclusion (Online Resource 3). Finally, six articles published between 1969 and 2020 fulfilled the inclusion criteria and thus remained for analysis [16-21]. All included studies were case series without controls.

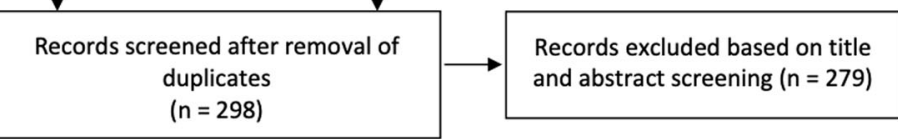

Full-text articles excluded $(n=13)$

Main reasons for exclusions:

- Number of patients $<10(n=8)$

- No presentation of periodontal problems $(n=4)$

- Review article $(n=1)$

\section{Summary of study characteristics}

\section{Population}

The studies included a total of 1278 patients (Table 1). In one study, 1120 records of LCH patients were screened for patients with oral symptoms and subsequently, the data of 114 patients presenting oral involvement were included and presented [18]. Thus, detailed data were available from six studies with a total of 272 patients.

All studies provided data about the proportion of males and females; i.e., out of 272 patients, 94 (35\%) were females and $178(65 \%)$ males. The age of the patients ranged between 2 weeks and 73 years at the time of $\mathrm{LCH}$ diagnosis. Three studies provided the mean age of patients that amounted to $1.7,4.8$, and 37.3 years, respectively [19-21].

A total of 131 patients (48\%) suffered from a singlesystem disease pattern or eosinophilic granuloma, and 137 patients $(50 \%)$ were diagnosed with a multi-system disease pattern or disseminated histiocytosis, Hand-SchüllerChristian, or Letterer-Siwe disease. In four patients (2\%), disease pattern was not specified [16]. 


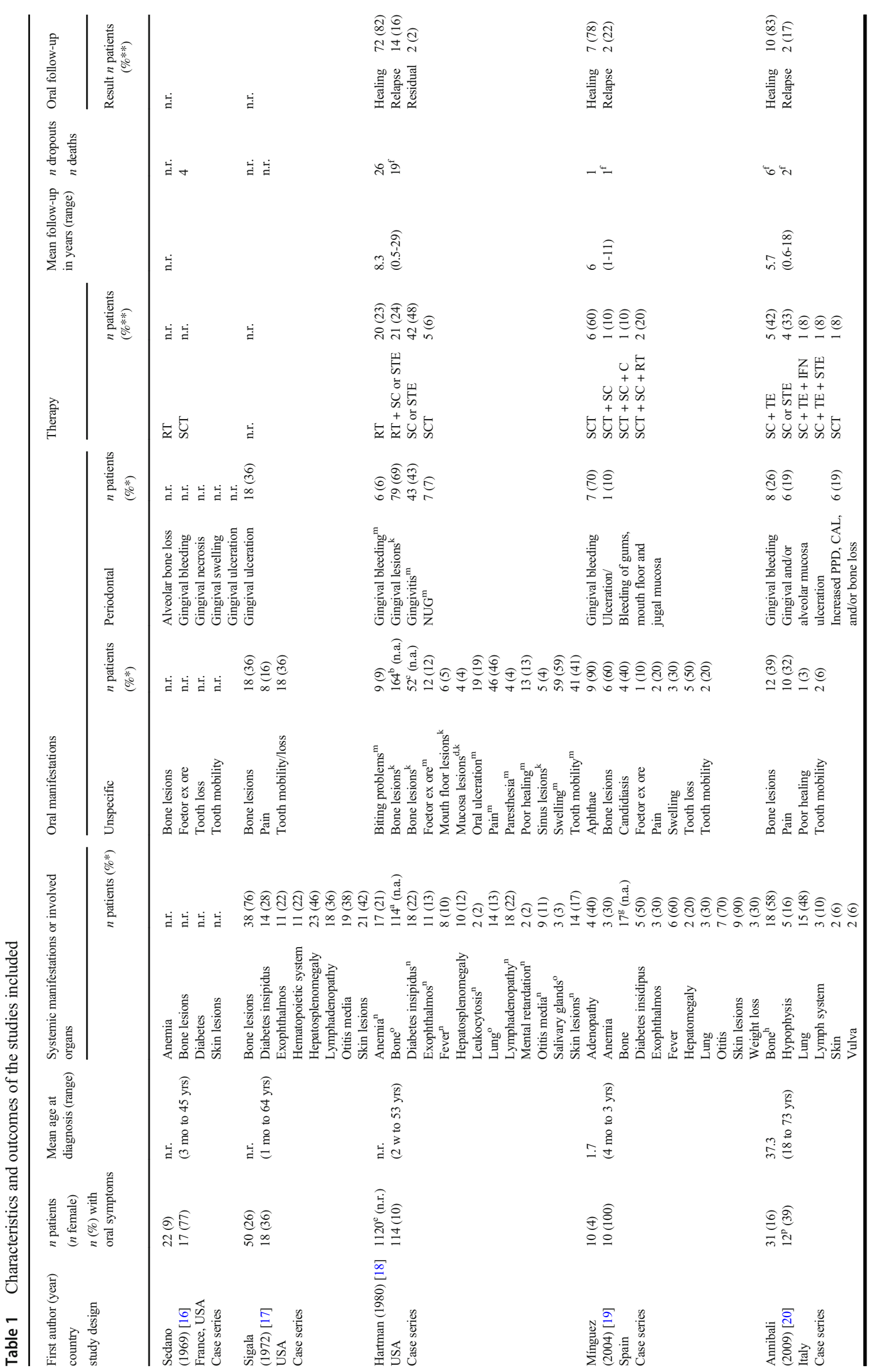




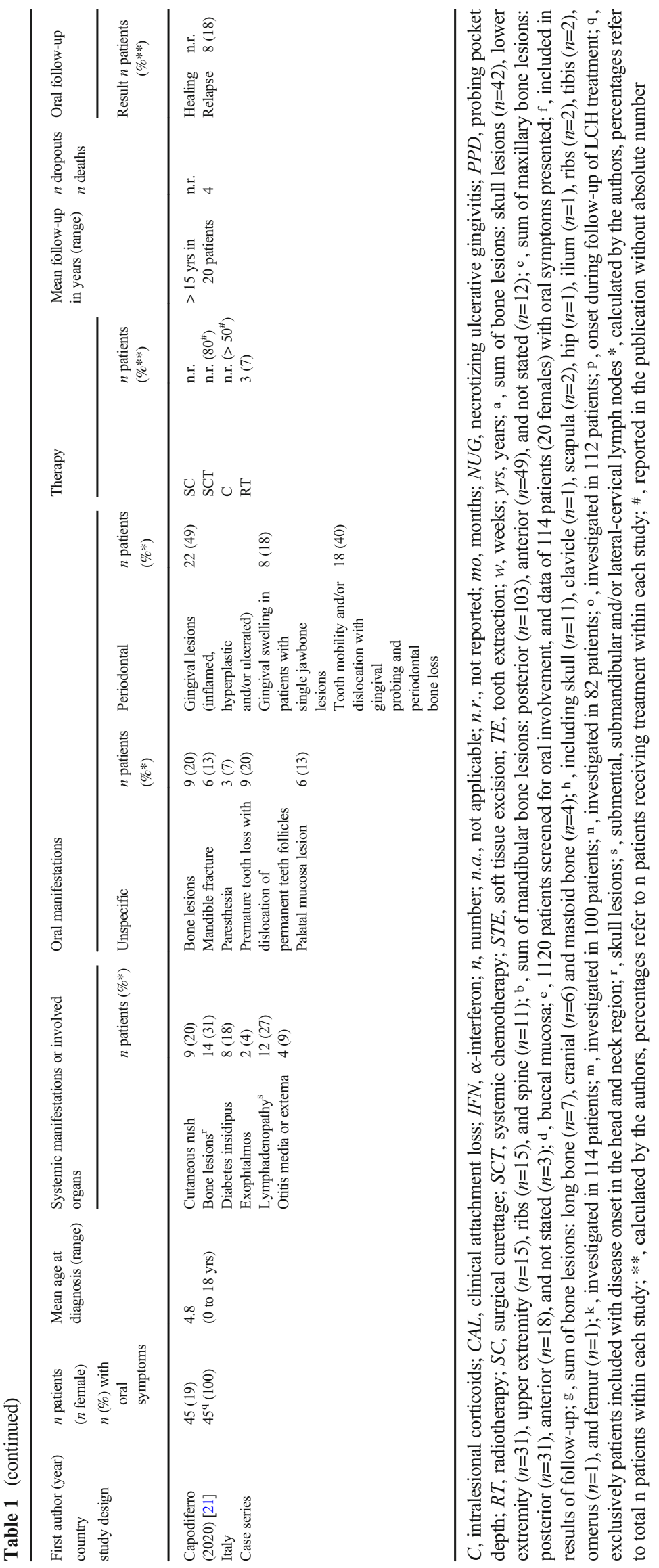


In all studies, systemic manifestations were described (Table 1). Involvement of the skeletal system was documented as extraoral bone lesions in 70 out of 126 patients ( $56 \%$ ) [17, $20,21]$. In addition, two studies differentiated between various bone types involved $[18,19]$. Subsequent to bone lesions, the involvement of the following three organs and/or symptoms was most frequently reported: skin or skin lesions (55 out of 218 patients, $25 \%$ ), hypophysis or diabetes insipidus (50 out of 218 patients, $23 \%$ ), and lymph system or lymphadenopathy (43 out of 173 patients, $25 \%$ ). Additional organs affected and/or related symptoms are shown in Table 1.

\section{Primary outcome: periodonal manifestations}

A total of 216 out of 1278 patients (17\%) demonstrated oral involvement (Table 1). Periodontal diagnoses were gingivitis (43 out of 100 patients, $43 \%$ ) and necrotizing ulcerative gingivitis (7 out of 100 patients, $7 \%$ ). In addition, periodontal findings comprised gingival bleeding (21 out of 141 patients, $15 \%$ ), gingival ulcerations or lesions (126 out of 199 patients, $63 \%$ ), gingival swelling ( 8 out of 45 patients, 18\%), and increased pocket depths and/or clinical attachment loss (24 out of 76 patients, $32 \%$ ).

The most commonly reported oral symptoms, not unambiguously related to periodontal inflammation, included tooth loss and/or tooth mobility (76 out of 236 patients, $32 \%$ ), oral pain (66 out of 191 patients, $35 \%$ ), and swelling (62 out of 110 patients, $56 \%$ ). Alveolar bone lesions were found in 45 out of 136 patients $(33 \%)$. In addition, one study differentiated between the regions of bone lesions and observed most lesions in posterior regions of the mandible (103 out of 114 patients, 90\%) [18]. Further oral symptoms are presented in Table 1.

\section{Secondary outcome: treatment modalities and response to treatment}

Three of the included studies reported the number of patients receiving systemic and/or local treatment measures [18-20]. In one of these studies, patients were already receiving a systemic chemotherapy when oral symptoms were detected [20]. These patients received an additional second-line therapy. Treatment measures included systemic chemotherapy (12 out of 110 patients, $11 \%$ ) with vinblastine, corticoids, and/or methotrexate; radiotherapy ( 20 out of 110 patients, $18 \%$ ); oral surgery ( 52 out of 110 patients, $47 \%$ ) including curettage, soft tissue excision, and/or tooth extractions; or a combination of these therapeutic approaches (26 out of 110 patients, $24 \%$ ).

The mean duration of follow-up was given in three out of six studies and varied from 6 months to 29 years [18-20]. The mean observation period amounted to $5.7,6$, and 8.3 years, respectively. Dropouts were reported in three out of six studies [18-20]. The main reason for dropouts was a too-long distance between residence and study center and thus decision for a nearer clinic for follow-up in six patients [20]. In 27 patients, the reasons for dropouts were not provided [18, 19]. Additionally, 30 patients died during follow-up [16, 18-21]. In 16 patients, death was related to $\mathrm{LCH}$, while 14 patients died due to other or unknown reasons.

In 89 out of 109 patients ( $82 \%$ ), healing after therapeutic interventions of oral lesions was documented during followup [18-20]. While 26 out of 154 patients (17\%) developed new oral manifestations, two patients $(2 \%)$ showed residual oral lesions.

\section{Quality assessment}

The assessment of the risk of bias of the included case series is illustrated in the Online Resource 5 and was based on the checklist from Moga et al. [23] to evaluate the methodological and reporting quality of case series. Percentage of positively graded items relevant for quality assessment ranged from 24 to $65 \%$; i.e., high to moderate risk of bias was present in all included case series.

\section{Discussion}

The purpose of the present systematic review was to assess periodontal manifestations and treatment modalities in $\mathrm{LCH}$ patients. Six case series with a total of 1278 patients suffering from LCH were included, and absolute and relative frequencies of symptoms calculated. The frequency of oral symptoms was heterogenous and ranged from 10 to $100 \%$. In 216 patients, oral symptoms were reported. Oral lesions affected both hard and soft tissues. Periodontal findings comprised most frequently gingival diseases, i.e., gingival ulcerations or lesions and gingival bleeding. Increased pocket depths and attachment loss were reported in two case series. The remaining oral findings were mostly unspecific with a possible, but not mandatory, relation to periodontal diseases. Among these were with varying frequencies of oral pain, oral swelling, tooth loss or tooth mobility, foetor ex ore, and biting problems. Bone lesions occurred most frequently and affected predominantly the posterior regions of the mandible. From a radiographic point of view, a similarity of bone lesions to a wide spectrum of diseases was described, such as odontogenic cysts, certain benign and malignant tumors, periapical lesions, and periodontal bone loss.

In the current classification of periodontal and peri-implant diseases and conditions, LCH was recently listed as group of "conditions which affect the periodontal apparatus independently of dental plaque/biofilm-induced inflammation" $[10$, 11]. The authors reported that LCH may lead to periodontal inflammation and severe periodontal destruction with increased pocket depths, alveolar bone loss, and tooth loss. The strength of association was estimated as moderate. 
Herein, we aimed to systematically evaluate the available evidence and identified six case series with pronounced heterogeneity regarding patient and disease characteristics and methodology. In this context, the following aspects may be discussed:

1. The age of patients in the included case series showed a wide range between 2 weeks and 73 years. In one study, $40 \%$ of patients were up to 9 years old [18], and in three studies, the majority of patients constituted children up to 6 years [16, 17, 19]. In contrast, Annibali et al. [20] included only patients $\geq 18$ years. These patients were already receiving active treatment for systemic disease control when oral examinations were performed.

2. Patients showed different disease stages and duration. In 64 patients, oral lesions were reported to present the first manifestation of $\mathrm{LCH}[16,17,19,21]$. In two studies, 22 out of 41 patients developed oral lesions after initial LCH diagnosis and under systemic treatment $[19,20]$. The length of follow-up varied between 6 months and 29 years in the included case series. LCH may clinically manifest itself in several acute phases and oral symptoms may newly develop or return in the course of disease. Thus, oral lesions may constitute a first sign of initial disease manifestation or a sign of disease reactivation.

3. LCH is typically characterized by a very heterogeneous clinical picture [8]. In the studies included, patients also presented different disease severities and patterns, as revealed by a wide range of systemic comorbidities and complications. While $131(48 \%)$ patients suffered from a single-system disease, $137(50 \%)$ were diagnosed with a multi-system disease pattern. Sedano et al. [16] found oral lesions as the only sign of disease in three patients. In contrast, all patients assessed by Minguez et al. [19] demonstrated a chronic disseminated form of LCH. The remaining studies included patients with varying disease patterns. In 16 patients, disease showed even a fatal course, and patients died following severe sequelae related to their underlying disease [16, 18-21]. Two studies differentiated between different $\mathrm{LCH}$ disease patterns when reporting on oral involvement $[18,20]$. One of these observed that unifocal oral involvement (one lesion) was associated with a multi-systemic disease pattern while multifocal oral lesions (> 1 lesion) were related to unisystemic disease [20]. However, there was no explanation for this association.

4. The case series included showed a moderate to high risk of reporting bias (Online Resource 5). Outcome parameters were mainly not predefined and reported for the first time in the results section of the studies. Thus, it seems difficult for the reader to distinguish between findings not investigated and findings investigated, but not detected and therefore not reported. To address this, we considered findings not reported in any patient as not investigated. The reporting frequency of different oral symptoms varied. While gingival ulceration was evaluated in all included case series (272 patients), probing pocket depths and clinical attachment loss were examined in two studies (76 patients) $[20,21]$. Consequently, the reference number of patients was adapted for each symptom when absolute or relative frequencies were calculated. A further source of bias may be the different sizes of included case series, ranging from 10 patients [19] to 1120 patients [18]. Out of the 1120 patients, however, only the data of patients with oral symptoms were presented in detail, i.e., of 114 patients.

5. In addition, severity and/or quantification of outcome parameters were not reported. For example, tooth loss was described in four studies [16, 17, 19, 21]. However, no data on the number of missing teeth were available. Similarly, Annibali et al. [20] recorded probing pocket depths and clinical attachment loss in 6-month intervals and summarized findings as periodontal damage found in six out of 31 patients.

6. Finally, no details were provided on the clinical and/or radiological examination methods used to assess outcome parameters. In one study, patients were assessed by different clinicians at follow-up examinations [20]. Information about examiner calibration was lacking. Thus, the reproducibility and reliability of applied methods appear difficult to estimate.

Depending on the type and severity of oral lesions, patient quality of life may be significantly impaired [25]. Four out of six studies reported on oral pain. Sigala et al. [17] described pain as the chief complaint in eight out of 18 patients with oral symptoms. Furthermore, $42 \%$ of patients complained of negative outcomes on quality of life due to oral lesions [20].

The reported treatment for oral lesions was surgical curettage of bone lesions, associated with tooth extractions and/or excision of involved soft tissues. The prognosis was favorable and most lesions disappeared, as evidenced by the overall remission rate of $82 \%$. Non-surgical periodontal treatment was not reported in any of the included case series. As LCH was described to affect the periodontal tissues independently of dental plaque/ biofilm-induced inflammation [10,11], mechanical biofilm removal, and/or scaling and root planning of diseased periodontia may be not expected as first-line therapy of oral lesions. Nevertheless, in case of periodontal inflammation, we suggest that oral treatment may be supported by measures of periodontal infection control, e.g., oral hygiene instruction and biofilm management and complemented by regular periodontal controls.

\section{Conclusions}

Based on the evidence from the case series with at least 10 patients, oral symptoms including periodontal 
manifestations typically seen for periodontal diseases may occur as initial and/or long-term symptoms and with varying frequencies $(10-100 \%)$ in patients with LCH. Thereby, LCH may mimic more common pathologies such as periodontal diseases. A thorough clinical inspection of the oral cavity for affected patients including the periodontal tissues is mandatory to detect any oral manifestation at an early stage. In case of oral and periodontal symptoms not or hardly explainable by other factors (e.g., insufficient oral hygiene) and/or a lack of response to conventional treatments, dentists should generally consider the possibility of an underlying systemic disease and initiate further diagnostic procedures by referral to a specialist.

Supplementary Information The online version contains supplementary material available at https://doi.org/10.1007/s00784-021-03873-0.

Acknowledgements Parts of this research were conducted by S. A. Bernauer in partial fulfillment of the requirements for a DDS degree from the University of Basel, Switzerland.

Some aspects of this work were presented as a poster (Bernauer SA, Walter C, Schmidt JC: Periodontal manifestations of Langerhans cell histiocytosis - a systematic review. SSP annual conference September 12-13, 2019, Baden, Switzerland).

Funding Open Access funding provided by Universität Basel (Universitätsbibliothek Basel). The study was self-funded by the authors and their respective institutions.

\section{Declarations}

Ethical approval Not applicable.

Informed consent Not applicable.

Conflict of Interest The authors Julia C. Difloe-Geisert, Selina A. Bernauer, Noémie Schneeberger, Michael M. Bornstein, and Clemens Walter all declare that they have no conflict of interest.

Open Access This article is licensed under a Creative Commons Attribution 4.0 International License, which permits use, sharing, adaptation, distribution and reproduction in any medium or format, as long as you give appropriate credit to the original author(s) and the source, provide a link to the Creative Commons licence, and indicate if changes were made. The images or other third party material in this article are included in the article's Creative Commons licence, unless indicated otherwise in a credit line to the material. If material is not included in the article's Creative Commons licence and your intended use is not permitted by statutory regulation or exceeds the permitted use, you will need to obtain permission directly from the copyright holder. To view a copy of this licence, visit http://creativecommons.org/licenses/by/4.0/.

\section{References}

1. Guyot-Goubin A, Donadieu J, Barkaoui M, Bellec S, Thomas C, Clavel J (2008) Descriptive epidemiology of childhood Langerhans cell histiocytosis in France, 20002004. Pediatr Blood Cancer 51:71-75
2. Salotti JA, Nanduri V, Pearce MS, Parker L, Lynn R, Windebank KP (2009) Incidence and clinical features of Langerhans cell histiocytosis in the UK and Ireland. Arch Dis Child 94:376-380

3. Stalemark H, Laurencikas E, Karis J, Gavhed D, Fadeel B, Henter JI (2008) Incidence of Langerhans cell histiocytosis in children: a population-based study. Pediatr Blood Cancer 51:76-81

4. Baumgartner I, von Hochstetter A, Baumert B, Luetolf U, Follath F (1997) Langerhans'-cell histiocytosis in adults. Med Pediatr Oncol 28:9-14

5. Arico M, Girschikofsky M, Genereau T et al (2003) Langerhans cell histiocytosis in adults. Report from the International Registry of the Histiocyte Society. Eur J Cancer 39:2341-2348

6. Allen CE, Ladisch S, McClain KL (2015) How I treat Langerhans cell histiocytosis. Blood 126:26-35

7. Collin M, Bigley V, McClain KL, Allen CE (2015) Cell(s) of Origin of Langerhans Cell Histiocytosis. Hematol Oncol Clin North Am 29:825-838

8. Abla O, Egeler RM, Weitzman S (2010) Langerhans cell histiocytosis: current concepts and treatments. Cancer Treat Rev 36:354-359

9. Emile JF, Abla A, Fraitag S et al (2016) Revised classification of histiocytoses and neoplasms of the macrophage-dendritic cell lineages. Blood 127:2672-2681

10. Albandar JM, Susin C, Hughes FJ (2018) Manifestations of systemic diseases and conditions that affect the periodontal attachment apparatus: case definitions and diagnostic considerations. J Clin Periodontol 45(Suppl 20):S171-S189

11. Jepsen S, Caton JG, Albandar JM et al (2018) Periodontal manifestations of systemic diseases and developmental and acquired conditions: consensus report of workgroup 3 of the 2017 World Workshop on the Classification of Periodontal and Peri-Implant Diseases and Conditions. J Clin Periodontol 45(Suppl 20):S219 S229

12. Moher D, Liberati A, Tetzlaff J, Altman DG, Group P (2009) Preferred reporting items for systematic reviews and meta-analyses: the PRISMA statement. PLoS Med 6: e1000097

13. Liberati A, Altman DG, Tetzlaff J et al (2009) The PRISMA statement for reporting systematic reviews and meta-analyses of studies that evaluate health care interventions: explanation and elaboration. PLoS Med 6:e1000100

14. Walter C, Schmidt JC, Rinne CA, Mendes S, Dula K, Sculean A (2020) Cone beam computed tomography (CBCT) for diagnosis and treatmentt planning in periodontology: systematic review update. Clin Oral Investig 24:2943-2958

15. Landis JR, Koch GG (1977) The measurement of observer agreement for categorical data. Biometrics 33:159-174

16. Sedano HO, Cernea P, Hosxe G, Gorlin RJ (1969) Histiocytosis X. Clinical, radiologic, and histologic findings with special attention to oral manifestations. Oral Surg Oral Med Oral Pathol 27:760-771

17. Sigala JL, Silverman S Jr, Brody HA, Kushner JH (1972) Dental involvement in histiocytosis. Oral Surg Oral Med Oral Pathol 33: $42-48$

18. Hartman KS (1980) Histiocytosis X: a review of 114 cases with oral involvement. Oral Surg Oral Med Oral Pathol 49: $38-54$

19. Minguez I, Minguez JM, Bonet J, Penarrocha M, Sanchis JM (2004) Oral manifestations of chronic disseminated histiocytosis. A report of 10 cases. Med Oral 9:152-154

20. Annibali S, Cristalli MP, Solidani M et al (2009) Langerhans cell histiocytosis: oral/periodontal involvement in adult patients. Oral Dis 15:596-601 
21. Capodiferro $\mathrm{S}$, Tempesta A, Limongelli L, Ingravallo G, Maiorano E, Sfasciotti GL, Bossu M, Polimeni A, Favia G (2020) Primary oro-facial manifestations of Langerhans cell histiocytosis in pediatric age: a bi-institutional retrospective study on 45 cases. Children $7: 104$

22. Schmidt JC, Walter C, Rischewski JR, Weiger R (2013) Treatment of periodontitis as a manifestation of neutropenia with or without systemic antibiotics: a systematic review. Pediatr Dent 35:E54-E63

23. Moga C, Guo B, Schopflocher D, Harstall C (2012) Development of a quality appraisal tool for case series studies using a modified Delphi technique. Institute of Health Economics (IHE), Edmonton
24. Mendes S, Rinne CA, Schmidt JC, Dagassan-Berndt WC (2020) Evaluation of magnetic resonance imaging for diagnostic purposes in operative dentistry - a systematic review. Clin Oral Investig 24: $547-557$

25. Buset SL, Walter C, Friedmann A, Weiger R, Borgnakke WS, Zitzmann NU (2016) Are periodontal diseases really silent? A systematic review of their effect on quality of life. J Clin Periodontol 43:333-344

Publisher's note Springer Nature remains neutral with regard to jurisdictional claims in published maps and institutional affiliations. 